\title{
The Asymmetric Magnets Problem
}

\author{
Brian Weatherson
}

There are many controversial theses about intrinsicness and duplication. The first aim of this paper is to introduce a puzzle that shows that two of the uncontroversial sounding ones can't both be true. The second aim is to suggest that the best way out of the puzzle requires sharpening some distinctions that are too frequently blurred, and adopting a fairly radical reconception of the ways things are.

\section{Two Theses about Duplication}

In all of David Lewis's discussions of intrinsicness and duplication, he held that the two concepts are connected by a tight circle of interdefinition. Duplicates share all of their intrinsic features, and objects that share all of their intrinsic features are duplicates. (Lewis, 1983b,a; Langton and Lewis, 1998). Both of these claims are a little controversial. One might hold that some impure properties that aren't shared by all duplicates, like having George Clooney as a part, are nevertheless intrinsic since gaining or losing them seems to amount to a non-Cambridge change (Weatherson, 2006). And one might hold that some properties which don't differ between duplicates by definition, such as being a duplicate of the Louvre as it actually is, are nevertheless extrinsic (Dunn, 1990). So maybe Lewis's tight circle of interdefinition is not beyond question. But the following principle seems utterly uncontroversial to me.

\section{Intrinsicness Principle :}

- If $a$ and $b$ differ in their pure intrinsic features, they are not duplicates;

- If $a$ and $b$ have the same pure intrinsic features, then they are duplicates

That conjunction is the first of our (hitherto) uncontroversial theses. The second needs a bit more work to state formally.

It is fairly intuitive that whether two objects are duplicates is not an emergent feature of reality. In some sense, whether two complex are duplicates just depends on the properties of their parts and the relations between their parts. But this claim does turn out to be controversial; David Lewis (1983b) has controverted it. To a first approximation, his theory says that whether two objects are duplicates depends on whether they share the same perfectly natural properties. If there are any perfectly natural properties that are emergent, i.e. which are properties that complex objects have but not in virtue of the properties of or relations between their parts, then whether two objects are duplicates will also be emergent. Now Lewis doesn't think there are any emergent perfectly natural properties, since the existence of such

\footnotetext{
$\dagger$ Penultimate draft only. Please cite published version if possible. Final version published in Philosophical Perspectives 20 (2006): 479-92. Thanks to the Philosophy Program at the RSSS, ANU, where this was first drafted, to audiences at University of Manitoba and Stanford University, and to the attendees at my seminar on David Lewis at Cornell University. I am especially grateful to Ben Caplan, John Hawthorne, Ishani Maitra, Raul Saucedo and Wolfgang Schwarz.
} 
properties would be incompatible with the thesis of Humean Supervenience. But Lewis doesn't think that Humean Supervenience is a necessary truth, let alone a conceptual truth, but at best a contingent truth. So the principle that duplication is not emergent is not something that is true in virtue of the concept of duplication.

Still, nothing in Lewis's views suggest that the following principle is false. If all the fundamental properties are not emergent, i.e. they are properties that complex things have in virtue of the fundamental properties of and relations between their parts, then duplication is not emergent. We might try and formalise this as follows. If all the fundamental properties are not emergent, then if the parts of $x$ and $y$ are duplicates, then $x$ and $y$ are duplicates. This principle is, however, too strong. It doesn't account for the possibility that the parts of $x$ and $y$ are arranged differently. For instance, in the following example, the fusion of $a$ and $b$ is not a duplicate of the fusion of $c$ and $d$, even though $a$ is a duplicate of $c$ and $b$ is a duplicate of $d$.
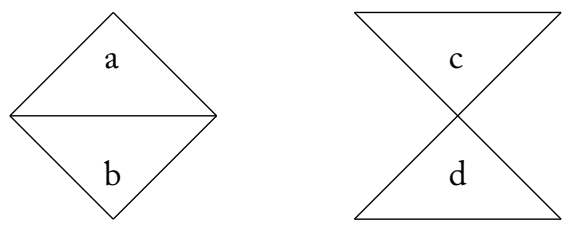

The problem is that the arrangement of the two objects is different. So what we need is a principle that says that if all the perfectly natural properties are not emergent properties, and if the parts of $x$ and $y$ are duplicates, and those parts are arranged the same way, then $x$ and $y$ are duplicates. Saying this formally is not exactly trivial. The following version uses the idea of an isometry ${ }^{1}$.

Parts Principle This principle holds in all worlds in which no fundamental properties or quantities are emergent. If $\mathrm{X}$ and $\mathrm{Y}$ are sets of material objects, $a$ is the fusion of the members of $\mathrm{X}$ and $b$ is the fusion of the members of $\mathrm{Y}, f$ is a function $\mathrm{X} \rightarrow \mathrm{Y}$, and $i$ is an isometry defined on the space that $\mathrm{X}$ and $\mathrm{Y}$ are in, and the following conditions hold:

- For all $x$ in $\mathrm{X}, f(x)$ is a duplicate of $x$; and

- For all $x$ in $\mathrm{X}$, if $r$ exactly occupied by $x$, then $i(r)$ is the region exactly occupied by $f(x)$,

then $a$ and $b$ are duplicates.

The Parts Principle is not as easy to state as the Intrinsicness Principle, but I think the idea it is expressing is fairly intuitive. Nevertheless, I think the two principles cannot both be true.

\footnotetext{
${ }^{1}$ An isometry is "a transformation that does not change the distance between points" (Yaglom, 1962, 11). That is, it is a function from points to points that doesn't change distances. Although the isometry is initially defined as a function on points, it can obviously be extended to a function from regions to regions. If $r$ is a region, i.e. a set of points, then $f(r)$ is $\{f(x): x \in r\}$.
} 


\section{Three Distinctions}

The problem I'll be focussing on looks rather simple, but it brings out several points that seem to have metaphysical interest. In particular, it highlights the importance of three distinctions that are easy to blur when doing metaphysics. It will make the exposition of the puzzle easier to place these distinctions up front.

The first distinction is between features and properties. Most metaphysicians accept that to fully characterise the world, we need to do more than say what exists. As well as saying what there is, we need to say how the things that exist are. It is easy to assume that to do that, we need to say what properties things have. But this need not be correct, or at least it need not be correct if we are looking to characterise the world in the most fundamental way. It might be that the fundamental features of reality are quantities, i.e. features that objects have to different degrees or in different amounts. Properties, like being green are features, but quantities, like mass or velocity are also features, just features that can be instantiated to different degrees or magnitudes. So feature is a more general category than property, and so as to not beg any questions, I'll talk about intrinsic features and fundamental features rather than intrinsic properties and fundamental properties throughout. My solution to the problem will involve assuming that at least some of the fundamental features of reality are indeed quantities not properties.

The second distinction is between fundamental features and perfectly natural features. Fundamental features are features that do not obtain in virtue of other features obtaining. The fundamental features are part of a minimal basis we need for characterising reality. Generally fundamental features are related to other fundamental features by exceptionless laws, though this is not part of their definition. What is definitional is that they are basic and that they provide a basis for characterising the world without redundancy. (As a Humean, I'd also say that there are no necessary connections between distinct fundamental features, but that is a controversial metaphysical thesis, not a defining characteristic.) Perfectly natural features are features that make for primitive objective resemblance between things that instantiate them. By a primitive objective resemblance, I mean an objective resemblance that does not obtain in virtue of sharing more basic (in the limit, fundamental) properties. David Lewis (1983b) assumes, without much by way of argument as far as I can see, that the fundamental features are the perfectly natural features. My solution to the problem will involve rejecting that identity.

The final distinction is between the thesis that all the fundamental non-spatiotemporal features of reality are intrinsic properties of points, and the thesis that these features are local features. Jeremy Butterfield (2006) has stressed the importance of this distinction for metaphysics. A feature is local to a point iff it is intrinsic to arbitrarily small regions around that point. For example, the slope of a curve at a point is local to that point, even though it isn't intrinsic to the point. So locality and intrinsicness can come apart. (This raises interesting questions about, for example, whether it is best to state the thesis of Humean Supervenience in terms of local properties or in terms of intrinsic properties of points.) I'll say more about the importance of this distinction in section 5 . 
I've already made use of these distinctions in setting out the principles about intrinsicness in section one. (In particular, it is crucial that the Parts Principle is stated in terms of fundamental rather than perfectly natural features.) Using them we can get to our central puzzle, the Asymmetric Magnets Problem.

\section{The Asymmetric Magnets Problem}

Our puzzle is similar to the spinning sphere, often thought to raise a problem for Humean Supervenience (Armstrong, 1980). The similarity is not in respect of its target; the puzzle is meant to be a puzzle for everyone who accepts those two principles, not just the Humean. Rather, the similarity is in that the puzzle is set in a world where there are homogeneous physical objects. Such a world is in many ways quite distant from actuality. But I think such worlds are useful fictions for elucidating the conceptual connections between central concepts in metaphysics. The puzzle is also set in a world with Euclidean spatial geometry. Again this is a fiction, but a useful one for working out conceptual connections.

In this world, some of the fundamental features are what we'll call vector features. (This is a much smaller deviation from actuality.) Vector features are either quantities like velocity the value of which is a vector, or properties like having velocity $v$, where $v$ is some vector. In particular, the strength and direction of the magnetic field throughout the world is a fundamental feature of the world. I'll assume that both space-time regions and physical objects can have these vector features, although I'm only going to focus on the field strength and direction at a point in a physical object. Finally, I'll assume that all of the fundamental physical quantities in the world are local. So there are no fundamental emergent quantities in the world. It might be worried that the last two assumptions are inconsistent, and that vector quantities could not be local. I'll come back to this worry in section 5 .

Some of the things in this world are magnets. These are homogeneous objects with a uniform non-zero magnetic field throughout. I'm going to represent the magnetic field strength and direction of such a magnet with an arrow pointing towards the north pole of the magnet. The length of the arrow is proportional to the strength of the field.

The simplest kind of magnet is a bar magnet, just like the kind I used to play with in primary school. (Apart from being homogeneous of course!) These are cuboids with equal heights and depths, and a long length in the direction of their magnetic field. Suzy is playing with some magnets and, tiring of using her magnets to grab the other childrens', decides to sharpen one end of each of her magnets for use as a weapon. The teacher notices this, confiscates the weaponised magnets, and lays them out on her desk. Here is what they look like from the teacher's point of view.

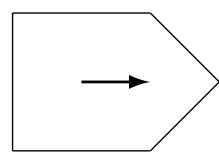

A

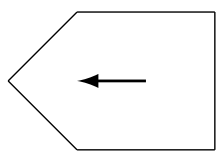

B

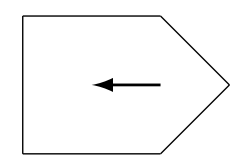

C 
I've added the labels.

Each magnet has one sharp end and one flat end. Each also has one north pole and one south pole. And, of course, each has one end to the right (from the teachers' point of view) and one end to the left. The distribution of these properties of ends is different in the three cases.

- A's north pole is sharp and to the right.

- B's north pole is sharp and to the left.

- C's north pole is flat and to the left.

Question: Which of the magnets are duplicates?

Answer: A and B, but not C.

I hope you agree with the answer! If not, let me provide a small argument.

A and B are intrinsic duplicates because we could 'line up' A and B by picking A up, spinning it around, and moving it across a bit. And that's only possible if the two objects are duplicates. This idea, that objects that can be transformed into one another by simple geometric transformations such as rotation and translation is a very deep part of our conceptual scheme. Consider, for example, Euclid's proof of proposition 4.

Let $A B C, D E F$ be two triangles having the two sides $A B, A C$ equal to the two sides $D E, D F$ respectively, namely $A B$ to $D E$ and $A C$ to $D F$, and the angle $B A C$ equal to the angle $E D F$. I say that the base $B C$ is also equal to the base $E F$, the triangle $A B C$ will be equal to the triangle $D E F \ldots$ For, if the triangle $A B C$ be applied to the triangle $D E F$, and if the point $A$ be placed on the point $D$ and the straight line $A B$ on $E$, then the point $B$ will also coincide with $E$, because $A B$ is equal to $D E$. Again, $\mathrm{AB}$ coinciding with $\mathrm{DE}$, the straight line $\mathrm{AC}$ will also coincide with $\mathrm{DF}$, because the angle $\mathrm{BAC}$ is equal to the angle EDF; hence the point $\mathrm{C}$ will also coincide with the point $\mathrm{F}$, because $\mathrm{AC}$ is again equal to DF. But $\mathrm{B}$ also coincided with $\mathrm{E}$; hence the base $\mathrm{BC}$ will coincide with the base $\mathrm{EF} \ldots$ and will be equal to it. Thus the whole triangle $\mathrm{ABC}$ will coincide with the whole triangle $\mathrm{DEF}$, and will be equal to it. (Euclid, 1956, 247-8)

As many mathematicians have pointed out over the centuries, this is not Euclid's finest moment as a geometer. The idea he's pushing is clear enough. If $A B C$ and $D E F$ satisfy the assumptions, then you can pick up $A B C$ and place it on $D E F$, so that the sides and vertices all coincide. Does this prove that the sides and angles in the original triangle are equal? Not really, or at least not without the assumption that picking up $A B C$ and moving it around doesn't change its side lengths or angle magnitudes. And Euclid hadn't said anything at that stage of the Elements to justify this assumption.

So qua axiomatic geometer Euclid has blundered here. But there is more to life than axiomatic geometry. There is, for instance, metaphysics. And the assumption Euclid is using here is, I think, a sound metaphysical intuition. (If it weren't, the 
complaints about this fundamental proof in Euclid would have been earlier, and more frequent, than they actually were.) That intuition is, I think, that intrinsic properties are not, ceteris paribus, changed merely by moving objects around. Of course other things are not always equal; the intrinsic properties of a car are not preserved if you drive it into a wall. But the kind of abstract motion that Euclid is contemplating when he moves $A B C$ onto $D E F$, or that I'm contemplating when I think about moving $B$ around so it lines up with $A$, does not destroy intrinsic properties. So that's an argument that A and B are intrinsically alike.

On the other hand, $\mathrm{A}$ and $\mathrm{B}$ each have a property that $\mathrm{C}$ lacks. Their magnetic field points towards their sharp end. This is in some sense a relational property, it is defined in part in terms of two things pointing in the same direction, but it doesn't seem like a relation between the magnet and anything else. In general, properties that things have in virtue of relations between their parts are intrinsic properties. (It is intrinsic to the earth, for example, that more of its surface is wet than dry, even though this property is defined in terms of a relation.) So this is an intrinsic property of A. And, given the plausibility of the Intrinsicness Principle, that's a reason to think that $\mathrm{A}$ and $\mathrm{C}$ are not intrinsic.

\section{The Principles and the Problem}

Here then is our problem. Try to answer the following question given the two principles: Is the direction of a vector feature an intrinsic feature of its bearer or not? If yes, then $\mathrm{A}$ and $\mathrm{B}$ are not duplicates. If no, then $\mathrm{A}$ and $\mathrm{C}$ are duplicates. (In fact all three are duplicates, though I won't prove this.) Neither way does it turns out that $A$ and $B$ are duplicates, but $C$ is not, as we need. The aim of this section is to spell out that little argument in more detail, so we can see how the principles relate to the problem.

First, we'll assume that the direction of a vector feature is an intrinsic feature of its bearer. We need a way to rigidly denote directions, so we'll call the direction that the vector in $A$ points $d_{1}$, and the direction that the vector in $B$ points $d_{2}$. Since $d_{1} \neq d_{2}, A$ and $B$ differ in their intrinsic properties. By the second clause of the Intrinsicness Principle, it follows that $A$ and $B$ are not duplicates.

Second, we'll assume that the direction of a vector feature is not an intrinsic feature of its bearer. Now we want to show that B and C are duplicates. To do this we'll use the Parts Principle. All of the fundamental quantities are local, so the Parts Principle applies. Now let the members of $\mathrm{X}$ and $\mathrm{Y}$ be the point-sized parts of $\mathrm{A}$ and C. Let $l$ be the distance from the tip of the pointed end of A to the tip of the pointed end of $\mathrm{C}$. The isometry $i$ is a translation with length $l$ and direction $d_{1}$, i.e. a function that maps any point to the point that is distance $l$ away from it in direction $d_{1}$. This isometry maps A onto C. By the first clause of the Intrinsicness Principle, and the assumption that direction is not intrinsic, every point in $\mathrm{A}$ is a duplicate of any point in C. So by the Parts Principle, $\mathrm{A}$ and $\mathrm{C}$ are required.

The conclusion is that if we want to say that $\mathrm{A}$ and $\mathrm{B}$ are duplicates, but $\mathrm{A}$ and $\mathrm{C}$ are not duplicates, then we can't hold on to both the Intrinsicness Principle and the Parts Principle. 
I think we should give up the Parts Principle. In particular, we should say that the Parts Principle holds only if all the perfectly natural features of reality are local, and this might fail to hold even if all the fundamental features of reality are local. The need for the distinction between these possibilities is, I think, the main lesson of the problem. But before we get to that conclusion, I want to address an objection to the argument so far.

\section{Two Worries About Locality}

I can an imagine an objection to this argument along the following lines. In the setup of the problem, I said that some of the fundamental features of reality are vector-valued quantities. I also said that all of the fundamental features of reality are local. But these assumptions are inconsistent. Vector properties are not intrinsic properties of points. (Since we're trying to hold on to the Intrinsicness Principle, we have to accept this.) Hence they are not, in the salient sense, local features of reality.

I think this objection is sound all the way to the last step. As noted above, we need to distinguish between local properties and intrinsic properties of points. The distinction is common in mathematics, but has not been paid sufficient attention in metaphysics. Jeremy Butterfield's (2006) is an important exception, one that was very influential on this paper.

So it is fair to say that all fundamental features of reality are local if all the facts about the world supervene on facts about the distribution of fundamental features in arbitrarily small regions, plus facts about the spatiotemporal arrangement of those regions. And there is no reason to think that positing vector features as fundamental is inconsistent with the fundamental features being local in this sense. Butterfield argues, persuasively, that velocity properties in Newtonian mechanics are not intrinsic properties of points, but he stresses that this doesn't mean they are not local in this sense. He is focussing on velocity, and what he says doesn't immediately translate to all vector properties. (It matters to his argument, for example, that velocities are conceptually connected to the positions of objects at different times, in a way that, for example, magnetic fields are not.) But I think his conclusions are independently plausible. Indeed, the argument from isometry above is an argument for the very same conclusion. So the short version of my reply is to concede that once we've allowed vector properties as fundamental, we can't say that all the fundamental features of reality are intrinsic properties of points and spatiotemporal relations between them, but this is consistent with saying that all the fundamental features of reality are local.

Once we've said that, however, a different kind of objection becomes salient. It might be thought that if the fundamental features are intrinsic properties of regions not of points, the natural version of the Parts Principle is slightly weaker than as stated. In particular, we should focus our attention to cases where the sets $\mathrm{X}$ and $\mathrm{Y}$ consist of objects with positive size. Because this weakening flows naturally from the definition of locality, it doesn't look like an ad hoc weakening. However this weakening does not at the end of the day help to save the Parts Principle. That's because we can find a different way to divide up $\mathrm{A}$ and $\mathrm{C}$ into parts of positive size 
so that the Parts Principle still applies. A sketch of how we'll (start to) divide up A is here.

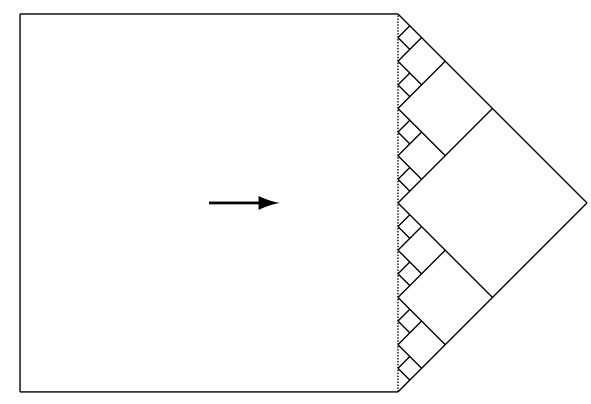

The idea is that we make one large square part, and then divide the rest of A up into infinitely many diamonds. We do this recursively. Note that we start with a triangle whose base is to the left and vertex to the right. We create from this a diamond whose four vertices are the vertex of the triangle, and the midpoints of each of the three sides of the triangle. If we imagine cutting this diamond out of the magnet, we'd be left with two small triangles, each with a base on the left and a vertex on the right. We can do the same trick to create diamonds and (in imagination) cut them out, leaving us with four triangles. Repeat this until we have an infinity of diamonds. The fusion of all these diamonds with the large square will be our original magnet. Moreover, since every part is symmetric around the axis perpendicular to $d_{1}$, each part will be a duplicate of the corresponding part in C. So the Parts Principle still tells us, falsely, that $\mathrm{A}$ and $\mathrm{C}$ are duplicates. We have to look somewhere else to avoid the problem.

\section{The solution and its problems}

The Asymmetric Magnets Problem looks easy. It is easy to say intuitively why A and $\mathrm{B}$ are duplicates, but $\mathrm{C}$ is not. The reason was given at the end of section three. In both $\mathrm{A}$ and $\mathrm{B}$, the magnetic field 'points' in the same direction that the physical object does, while in $\mathrm{C}$ this is not the case. The difficulty arises when we try and shoehorn this intuition into a formal theory. We need to say that it is intrinsic to the magnet that its magnetic field points the same way it does. And we need to say this without saying that the direction of the magnetic field is itself intrinsic. I know of one way to do this, but it involves some overheads. I'm not going to argue for this at any length here, but I think the difficulty of providing a general solution to the Asymmetric Magnets Problem is one of many reasons to think that we should learn to live with these overheads.

My solution starts with Lewis's definition of duplication. I gave a rough statement of this above; we now need a more precise statement. For Lewis, two objects are duplicates iff there is a mapping $m$ from parts of one to parts of the other that (a) is an isomorphism and (b) for all $n$-place perfectly natural properties $P$, and all parts $x_{1}, \ldots, x_{n}$ of the first object, $P x_{1} \ldots x_{n}$ iff $P m\left(x_{1}\right) \ldots m\left(x_{n}\right)$. So the objects are 
duplicates if their parts have the same natural properties, and stand in the same perfectly natural relations. That's how Lewis's theory goes; now we have to start adding variations. The first variation is quite radical, but one we have independent reason to make.

As John Hawthorne (2006) and David Denby (2001) have argued, Lewis's theory of properties has difficulties accounting for quantities. Hawthorne notes that if we just take individual mass properties, e.g. having mass $17 \mathrm{~kg}$, having mass $42 \mathrm{ng}$ etc as perfectly natural, there is no way to state physical laws involving mass, such as the law of gravitation, as simple statements where all predicates denote perfectly natural properties. But the theory of laws in Lewis (1983b) says that all physical laws are simple statements where all predicates denote perfectly natural properties. This is something of a problem. For different reasons, Denby suggests that we take determinables as being perfectly natural. The individual mass properties are perfectly natural, he suggests, but not fundamental. What is fundamental is the determinable, mass, of which they are determinate.

I think we should make a more radical move in the interests of simplicity. What reason do we have for thinking that the fundamental ways things are are properties rather than quantities or magntitudes? Very little reason, I'd say. Modern physics seems much more concerned with quantities than properties. What properties it is concerned with, such as being positively charged, seem to be derived from more fundamental quantities, such as charge. It would perhaps be convenient for formal semantics if the world had an object-property structure to match the subject-predicate structure of simple sentences. But we have no reason to believe the world will be so accommodating. It might turn out that there are a few fundamental quantities in the world. A quantity is a feature that objects have to different degrees. We can identify each value a quantity takes with a property. (Examples are properties like having mass $17 \mathrm{~kg}$.) But that shouldn't make us think that the properties are metaphysically primary. They might be derived from the quantities. Hawthorne's and Denby's arguments push us towards that conclusion, and I'll show here that assuming quantities are primary helps us state a solution to the Asymmetric Magnets Problem.

Some quantities take simple values. The values of the mass quantity, for instance, are sufficiently simple that they can be represented by real numbers. But not all quantities are like that. In some cases the values are structured entities, which are composed of a magnitude and some other some other part or parts. Vector quantities are like this. We can naturally think of vectors as structured entities composed of a direction and a magnitude. I'm going to assume, at least for the sake of solving this problem, that any perfectly natural quantity takes values that either are magnitudes (as mass does) or takes values that are structured entities composed, among other things, of a magnitude. (This is an empirical assumption, and it may well not be true. If it is not true, the analysis of duplication below will need to be made more complicated.) For ease of exposition, I'll say that a function $f$ is perfectly natural iff it maps objects onto values, such that there is some perfectly natural quantity such that for any $x, f(x)$ is the value that quantity takes with respect to $x$. So if the quantity is mass, $f(x)$ is $x^{\prime}$ 's mass. And I'll say that $|f(x)|$ is the magnitude of this value, in the 
sense described above. (The notation here is slightly non-standard, since I allow that magnitudes may be negative numbers. For example, if $f$ represents charge and $x$ is negatively charged, then $|f(x)|$ may be a negative number.)

Now for the definition of duplication. Two objects are duplicates iff there is a mapping $m$ from parts of one to parts of the other that (a) is an isomorphism and (b) for all $n$-place natural functions $f$, and all parts $x_{1}, \ldots, x_{n}$ of the first object, $\left|f\left(x_{1}, \ldots, x_{n}\right)\right|=\mid f\left(m\left(x_{1}\right), \ldots, f\left(m\left(x_{n}\right)\right) \mid\right.$. So the objects are duplicates if the magnitudes of each of the natural quantities of each of their parts are the same. This allows that the quantities can vary without loss of intrinsic character, provided there is no variation in magnitude.

The Asymmetric Magnets Problem suggests a view on which the directions of vector features are indirectly relevant to the intrinsic nature of objects. 'Indirectly' because changing the direction doesn't change the intrinsic properties of objects. But 'relevant' because the direction can matter, as we see when comparing $\mathrm{A}$ and $\mathrm{C}$. The definition of duplication in terms of quantities that take structured values allows us to capture this indirect relevance. We'll do so by defining a feature whose magnitude varies depending on how the object's shape and the direction of its vector features are coordinated.

Let $f$ be a function representing some perfectly natural quantity such that $f(x)$ is a vector. That is, $f$ represents some perfectly natural vector quantity. This quantity may or may not be fundamental, though it will be fundamental in the cases under consideration here. Let $c$ be a function that takes an object as input and returns its geometric centre as output. (By the geometric centre of $x$ I mean the centre of mass of an object with the same shape as $x$ and uniform mass density throughout.) Now suppose that the following function is perfectly natural.

$$
g(x, y, z)=d_{d f} \text { the cosine of the angle between } f(x) \text { and the ray from } g(y) \text { to } g(z)
$$

The motivation for taking this to be perfectly natural (but obviously not fundamental) is that it delivers the right results about the Asymmetric Magnets Problem, and it seems to deliver those results for the right reasons. To see it delivers the right results, just apply the above definition of duplication. Two objects are duplicates iff there is an isomorphism $m$ from the parts of one to the parts of the other such that for all $n$-place natural functions $f$, and all parts $x_{1}, \ldots, x_{n}$ of the first object, $\left|f\left(x_{1}, \ldots, x_{n}\right)\right|=$ $\mid f\left(m\left(x_{1}\right), \ldots, f\left(m\left(x_{n}\right)\right) \mid\right.$. To make the discussion easier, we'll redraw the magnets with some salient parts labelled.

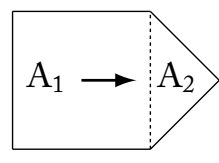

A

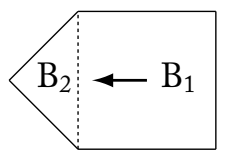

B

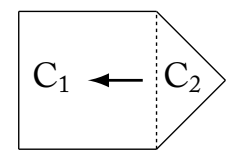

$\mathrm{C}$

Any isomorphism from $A$ to $B$ that satisfy this constraint has to map $A_{1}$ to $B_{1}$, and $A_{2}$ to $B_{2}$. And any isomorphism from $A$ to $C$ that satisfy this constraint has to map 
$A_{1}$ to $C_{1}$, and $A_{2}$ to $C_{2}$. Now let $f$ be the function whose value is represented by the arrow, and let $g$ be the function defined as above. So if $\mathrm{A}$ and $\mathrm{B}$ are duplicates, it must be the case that $g\left(\mathrm{~A}, \mathrm{~A}_{1}, \mathrm{~A}_{2}\right)=g\left(\mathrm{~B}, \mathrm{~B}_{1}, \mathrm{~B}_{2}\right)$. It is easy to verify that since $f(A)$ points in the same direction as the ray from the centre of $A_{1}$ to the centre of $A_{2}, g\left(A, A_{1}, A_{2}\right)=1$, and similarly the ray from the centre of $B_{1}$ to the centre of $B_{2}$ points in the same direction as $f(\mathrm{~B}), g\left(\mathrm{~B}, \mathrm{~B}_{1}, \mathrm{~B}_{2}\right)=1$. So there is no reason here to doubt that $\mathrm{A}$ and $\mathrm{B}$ are duplicates. On the other hand, since the ray from the centre of $\mathrm{C}_{1}$ to the centre of $\mathrm{C}_{2}$ is in the opposite direction to $f(\mathrm{C}), g\left(\mathrm{C}, \mathrm{C}_{1}, \mathrm{C}_{2}\right)=-1$. So there is no isomorphism from parts of $\mathrm{A}$ to parts of $\mathrm{C}$ that preserves the value of perfectly natural properties, so $\mathrm{A}$ and $\mathrm{C}$ are not duplicates, as required.

I don't doubt that there are other ways to solve this problem, so I certainly won't try arguing that this is the only solution. But I think it works, and the reason it works is because the values of natural quantities are structured entities, in this case vectors. Because they have structure, we can use one part of the structure (i.e. the magnitude) in determining what is directly relevant to intrinsicness, and another part of the structure (in this case the direction) in determining what is indirectly relevant. So it's an important advantage of using quantities rather than properties as the centrepiece of our metaphysics that the values of natural quantities can be structured entities, and having something like structured quantities seems crucial to solving this problem.

Although $g$ is represents a perfectly natural quantity, it does not represent a fundamental quantity. Instead, it represents a quantity whose value supervenes on the distribution of other perfectly natural quantities. So we have to allow that there is a distinction between the fundamental quantities and the perfectly natural quantities. I don't think this is a cost of the theory; there is no way to capture the idea that directions are indirectly relevant without distinguishing between the perfectly natural and the fundamental, so the Asymmetric Magnets Problem is a reason to make such a distinction. (I'm indebted here to Ben Caplan.)

We can reduce the apparent cost of this distinction by noting that one reason we might have for blocking redundant natural quantities does not apply here. (By a redundant quantity, I just mean one that supervenes on the fundamental quantities.) We don't want to say that disjunctive properties like being grue, that supervene on other natural properties, are perfectly natural. But that's not primarily because of the supervenience, but because of the fact that grueness doesn't make for resemblance amongst the things that instantiate it. So at least that reason for caring about redundancy doesn't apply here. (I'm indebted here to Raul Saucedo.)

Finally, it is crucial to my solution that $\mathrm{A}, \mathrm{B}$ and $\mathrm{C}$ have these parts. If $\mathrm{A}, \mathrm{B}$ and $\mathrm{C}$ are extended simples, I can't run the argument I make here. Indeed, if they are extended simples, it looks like they are duplicates by my definition. That seems bad. I think this is a problem that we don't need to worry about, because this isn't a real possibility. I'll concede for the sake of argument that there are such things as extended simples. What I don't see any need to concede the possibility of are asymmetric extended simples. In general, the way that we deduce that an object has parts is by noting it has different properties at different places. (This point is made 
in Sider (2003).) I think this is just the right strategy to use, as a matter of necessity. If an object has different properties in different locations, it has different parts in those different locations. So there could not be extended simples that are asymmetric magnets. The case where my theory produces the wrong result is an impossible case.

\section{Wrapping Up}

This paper has had several ambitions, some loftier than others. The most basic aim has been to introduce the Asymmetric Magnets Problem, and argue that it is going to be hard work for a systematic theory of intrinsicness to account for the facts about the problem. The more profound aims involve tearing apart concepts that metaphysicians often take for granted are interchangeable. My solution to the problem involves distinguishing local features from intrinsic features of points, fundamental features from perfectly natural features, and, most importantly, features from properties. The last of these is I think the biggest point. If we come to believe that quantities, not qualities, are the fundamental ways things are, then quite a bit of metaphysical orthodoxy needs rewriting. Some of that rewriting may be simple; just a matter of crossing out 'li' and writing in 'nt' in the middle of some words. But changes in fundamental metaphysics tend not to be isolated, and the rewriting project may lead to more wide-ranging changes. (Egan (2004) makes this point well, with an important illustration.) Now I certainly haven't given anything like a conclusive argument in this paper that we should set about that project immediately. I have, however, provided one reason to think the project will eventually be necessary, and I suspect that more reasons will be provided in the future.

\section{References}

Armstrong, David, (1980). “Identity Through Time.” In Peter van Inwagen (ed.), Time and Cause: Essays Presented to Richard Taylor, 67-78. Dordrecht: Reidel. (4)

Butterfield, Jeremy, (2006). "Against Pointillisme about Mechanics." British Journal for the Philosophy of Science 57: 709-753, doi:10.1093/bjps/ax1026. (3, 7)

Denby, David, (2001). “Determinable Nominalism." Philosophical Studies 102: 297-327, doi:0.1023/A:1010314926955. (9)

Dunn, J. Michael, (1990). "Relevant Predication 2: Intrinsic Properties and Internal Relations.” Philosophical Studies 60: 177-206, doi:10.1007/bf00367469. (1)

Egan, Andy, (2004). "Second-Order Predication and the Metaphysics of Properties." Australasian Journal of Philosophy 82: 48-66, doi:10.1080/713659803. (12)

Euclid, (1956). The Thirteen Books of the Elements, tr. Thomas L. Heath. New York: Dover. (5)

Hawthorne, John, (2006). "Quantity in Lewisian Metaphysics." In Metaphysical Essays, 229-237. Oxford: Oxford University Press. (9) 
Langton, Rae and Lewis, David, (1998). "Defining 'Intrinsic'." Philosophy and Phenomenological Research 58: 333-345, doi:10.2307/2653512. Reprinted in Papers in Metaphysics and Epistemology, pp. 116-132. (1)

Lewis, David, (1983). “Extrinsic Properties.” Philosophical Studies 44: 197-200, doi:10.1007/bf00354100. Reprinted in Papers in Metaphysics and Epistemology, pp. 111-115. (1)

-, (1983). "New Work for a Theory of Universals." Australasian Journal of Philosophy 61: 343-377, doi:10.1080/00048408312341131. Reprinted in Papers in Metaphysics and Epistemology, pp. 8-55. (1, 3, 9)

Sider, Theodore, (2003). "Maximality and Microphysical Supervenience." Philosophy and Phenomenological Research 66: 139-149, doi:10.1111/j.19331592.2003.tb00247.x. (12)

Weatherson, Brian, (2006). "Intrinsic Vs. Extrinsic Properties." In Edward N. Zalta (ed.), The Stanford Encyclopedia of Philosophy (Fall 2006 Edition). Metaphysics Research Lab, Stanford University. (1)

Yaglom, I. M., (1962). Geometric Transformations I. Random House: New York. (2) 